\title{
RESEARCH IN THERMOANEMOMETRIC MEASURING DEVICE OF PULSE FLOW OF TWO-PHASE MEDIUM
}

\author{
Vasyl Dmytriv ${ }^{1}$, Ihor Dmytriv ${ }^{1}$, Taras Dmytriv ${ }^{2}$ \\ ${ }^{1}$ Lviv National Agrarian University, Ukraine; ${ }^{2}$ Lviv Polytechnic National University, Ukraine \\ Dmytriv_V@ukr.net
}

\begin{abstract}
The scheme and peculiarities of the operation of the thermoanemometric measuring device of the twophase flow are considered on the example of milk transportation with air inclusion during the work of the milking machine. On the base of the thermal balance equation the mathematical model is developed that allows simulating the temperature of the sensor's perceptual elements and its mutual placement in the flow of the measured medium. The temperature impulse of the thermal field is measured; its intensity depends on the flow velocity and the ratio of "liquid-gas". The influence of the environment temperature and the initial temperature of the measured medium are eliminated. The sensors are located in a measured medium and do not distort the character of its movement. The results of modelling the parameters of the thermoanemometer and the data of experimental researches are analysed. In the automated milking machine the milk yield was measured by the thermoanemometer in the process of machine milking of cows. To determine the system of flow parameters of a two-phase medium, a single-chip microcontroller has been applied.
\end{abstract}

Keywords: thermoanemometer, two-phase flow, bridge circuit, current amplifier, impulse generator.

\section{Introduction}

Primary converters of thermoanomometric type are widely used in measuring the consumption of one-component media. The principle of thermoanemometric measurement of the mass flow of the medium is based on the influence of the flow of the liquid (in most cases, the gas flow) on the heat transfer of the heating element [1]. However, methods of thermoanomometric measurements are developed in the direction of improving the design of the sensors and their orientation in the measuring environment, and also there is a problem of sensor calibration [2]. The thermal flow sensor typically contains a thermoresistant element, which is heated by an electric current, which provides a temperature rise of the sensing element in relation to the measuring medium. A constant temperature difference between the perceiving element and the measured medium (flow of liquid or gas) is supported by the bridging scheme of activating the sensor, which provides a change in the heating power depending on the mass output of the measured medium. Another option of the thermoanemometer operation is that the thermal dispersion mass flowmeter operates with a constant sensor heating power, and the temperature difference varies depending on the mass flow rate of the measuring medium. The principles of work, design and application of industrial thermal mass flow meters are described by Olin J.G. [3].

The design of the sensor, the position in the flow and the nature of the flow affect the measurement interval of the mass loss of the thermoanomometric measuring device. Badarlis A. et al. optimized the position of the sensor in the flow and proposed the design of the perceptive element [4]. Baker R.C. and Gimson C. researched in the influence of the eccentricity of the element placement, construction and dimensions of the perceiving element [5]. The influence of the flow character in the measuring medium on the parameters of the sensor is analysed in the article of [6]. Klemen Rupnik et al. have developed a new measurement method for identifying the type of gas in a massive heat flow measuring device [7]. Artur Cebula analytically and numerically calculated the simulation results of temperature distribution along the heat flow measuring device [8]. A wide range of studies were carried out with the heat flow measuring device of mass output of gas by the Farzaneh-Gord M. et al. [9].

The nature of the transport of a two-phase medium (milk + air) in the milking machine is pulsating with a pulsation frequency corresponding to the pulsator frequency of the milking machine. The ratio of milk and air is variable in the process of milking and the nature of the increase and decrease in the speed of transportation is unstable. The mode of milk transporting by the milking machine is adequate to the regime of milk ejection of cows. Therefore, measuring the milk flow in the milking machine should correspond informatively to the real physiological process of milk ejection of the cow, and this should provide an adequate response of the milking machine. 
For the purpose of this task, a thermoanemometric measuring device of the two-phase pulsating flow is designed to measure the parameters of milk ejection and adaptation of the milking machine to the physiology of milk ejection of the cow. The measuring device does not distort information about the character of milk ejection [10].

\section{Materials and methods}

The construction of the thermoanemometric measuring device of the pulsating flow of a twophase medium contains a bridge circuit for measuring the voltage change and transforming it into a discrete-time digital signal. Thermistors operate in the mode of the given voltage imbalance of the bridge circuit. The imbalance in the voltage resulting from the heat removal is measured and the resistance of the elements due to changes in the quantity and velocity of the milk is changed. The scheme is designed so that the temperature of both ambient and milk do not affect the accuracy of the measurement (Fig. 1).

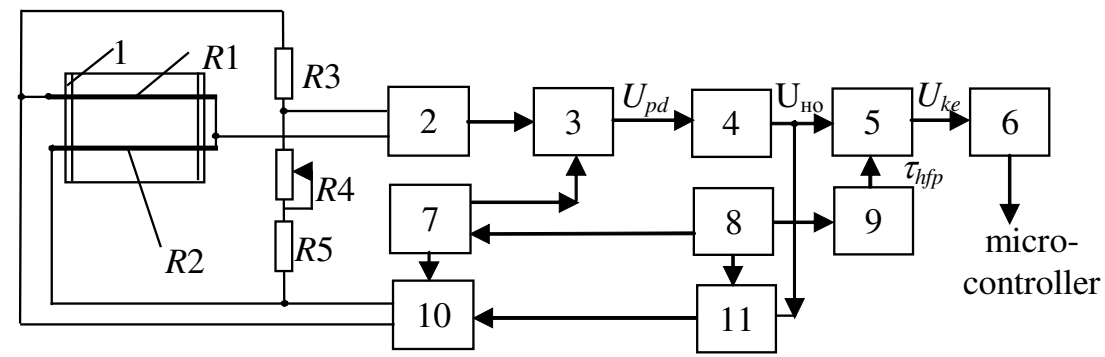

Fig. 1. Block scheme of thermoanemometric measuring device of pulsating flow of two-phase medium: 1 - body; $R 1, R 2$ - thermistor elements; $R 3, R 4, R 5$ - resistors of bridge circuit; 2 - alternating current amplifier; 3 - phase detector; 4 - null device; 5 - electronic key; 6 - bus-bar former; 7 - reference generator; 8 - power conditioner; 9 - high frequency pulser; 10 - rectangular oscillation generator, 11 - control unit

For thermistor sensor elements the material of sufficiently small heat conductivity is selected, which ensures low inertia, identity of thermal and temporal characteristics. The thermistor elements are half-arms of the bridge circuit with a four times difference in its electrical resistance. This ensures high sensitivity and independent operation of the electrical circuit, regardless of the ambient temperature and milk.

With the help of an automatic closed feedback system that includes a bridge circuit with thermistor sensors of $R 1, R 2$ and resistors of $R 3, R 4, R 5, \mathrm{AC}$ amplifier 2, phase detector 3 , null device 4 , control unit 11, rectangular oscillation generator 10 , the average value of the $\Delta t_{1}$ and $\Delta t_{2}$ overheat temperatures of the $R 1, R 2$ thermistor sensors is maintained above the ambient temperature. The difference between the maximum and the average value of the temperature of the elements (Fig. 2, b) is given by the transfer coefficient of the amplifying path and the zone of the sensitivity $\Delta U_{0}$ of the null device (Fig. 2, c). In the absence of milk $(v=0)$ (Fig. 2,a, $\left.q_{f}=0,0<\tau<\tau_{1}\right)$ the power taken from the thermistor elements is nonessential. The overheating temperature of $R 1$ and $R 2$ elements is maintained at the level of $\Delta t_{1}$ and $\Delta t_{2}$ (Fig. 2, b). Element heating is provided by a generator of rectangular oscillations, and the heating time is smaller compared to the cooling time $\left(\Delta \tau_{H O}<<\tau_{00}\right.$, Fig. 2, b, for example $\tau_{1}<\tau<\tau_{2}$ ). At the same time, the rate of voltage rise at the output of the phase detector is much higher than the velocity of its decrease. In a short heating time $\Delta \tau_{H O}$ of the elements, when the $U_{H O}$ output voltage of the 4 null device through the 5 electronic key is filled with highfrequency pulses (Fig. 2, e) from the generator 9, the certain number of pulses will go to the microcontroller through the 6 bus-bar former. In the presence of $q_{f}$ milk ejection in the sensor (Fig. 2, a, $q_{n} \neq 0$ ), the power output from the $R 1, R 2$ thermistor elements increases in proportion to the $v$ velocity in the $n$ power, where $n<1$. This leads to an increase in the duration of $\Delta \tau_{H 1}$ (Fig. 2, b) of the heating of the thermoresistor elements and its voltage supply with the amplitude $U_{H}$ from the 10 generator (Fig. 2, d). This will increase the number of pulses coming from the 9 pulse generator of high frequency through the electronic key 5 (Fig. 2, e) for the $\Delta \tau_{H}$ time. The principle of operation of the measuring device will be described analytically. 


\section{Development of the analytical model}

Due to the automatic system with a closed feedback, which includes blocks of $2,3,4,11,10$ and the bridge circuit in a state close to equilibrium, the equality (1) is fulfilled.

a)

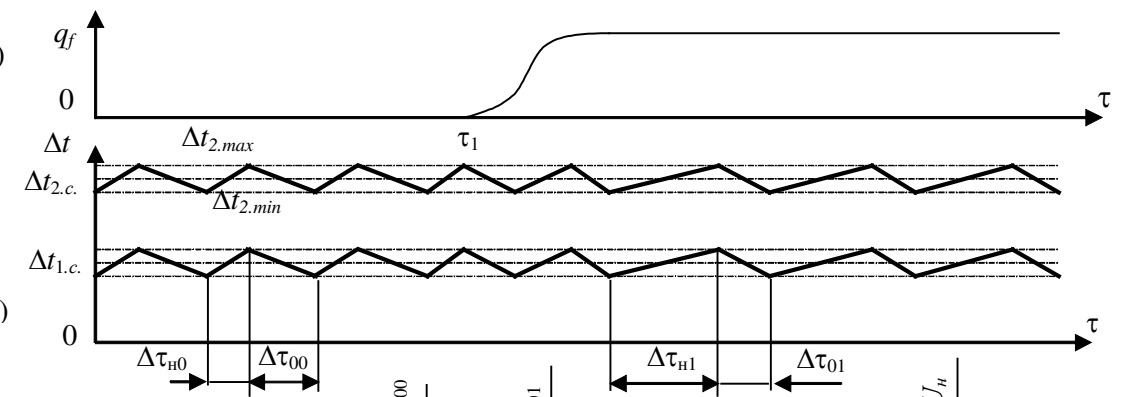

c)

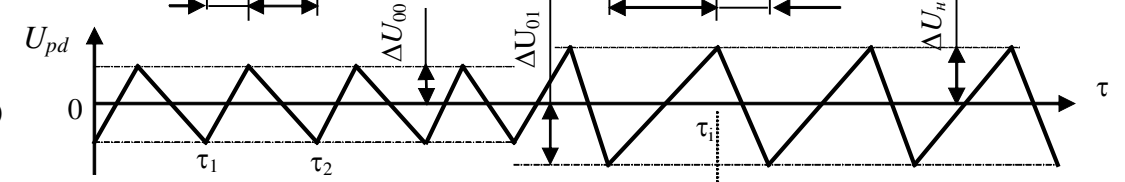

d)

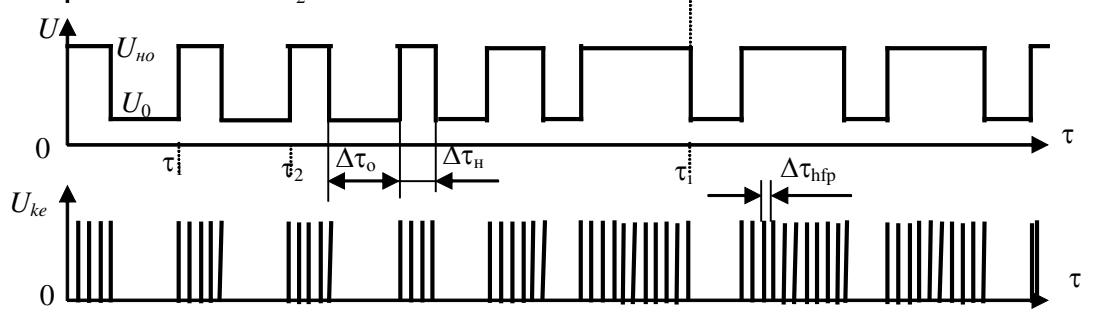

Fig. 2. Work diagrams of thermoanomometric counter of pulsating flow measuring device of

two-phase medium: $\mathrm{a}-q_{n}$ milk ejection; $\mathrm{b}-\Delta t_{1}$ and $\Delta t_{2}$ superheat temperature of thermistor elements; $c$ - output voltage of 3 phase detector and input voltage of 4 null device; $d$ - supply voltage of bridge circuit from the 10 square oscillation generator; $\mathrm{e}$ - characteristic of the output voltage of 5 electronic key

$$
R 1 \cdot(R 4+R 5)=R 2 \cdot R 3, R 8=(R 2 \cdot R 3 / R 1)-R 5,
$$

where $R 3, R 4, R 5$ - resistors of bridge circuit, as presented in Fig.1;

$R 1, R 2$ - resistors of thermistor elements with proportion of $R 1 / R 2=1 / 4$.

Increase of the $R 4$ resistance in the adjustment of the bridge scheme by dependence (2) is determined in the case of overheating of the $R 1$ and $R 2$ thermistor elements in relation to the temperature of milk.

$$
\Delta R 4=\frac{\partial R 4}{\partial R 2} \cdot \Delta R 2+\frac{\partial R 4}{\partial R 1} \cdot \Delta R 1 .
$$

Taking into account the equations (1) and (2), the dependence (3) is obtained.

$$
\Delta R 4=\frac{R 3}{R 1^{2}} \cdot(R 1 \cdot \Delta R 2-R 2 \cdot \Delta R 1) .
$$

Taking into account the (3) equation, the growth of the resistance of the thermoresistor elements with the change of its temperature of (4) and (5) the dependence (6) is obtained.

$$
\begin{gathered}
R 1=R 1_{0} \cdot\left(1+\alpha \cdot \Delta t_{1}\right), R 2=R 2_{0} \cdot\left(1+\alpha \cdot \Delta t_{2}\right), \Delta R 1=R 1_{0} \cdot \alpha \cdot \Delta t_{1}, \Delta R 2=R 2_{0} \cdot \alpha \cdot \Delta t_{2} . \\
\Delta t_{1}=t_{1}-t_{0}, \Delta t_{2}=t_{2}-t_{0} . \\
\Delta R 4=\frac{R 3 \cdot \alpha \cdot R 1_{0} \cdot R 2_{0}}{R 1^{2}} \cdot\left(\Delta t_{2}-\Delta t_{1}\right),
\end{gathered}
$$

where $R 1_{0}, R 2_{0}$ - accordingly, the resistance of the thermoresistive elements for a balanced bridge scheme at the $t_{0}$ cooling temperature; 
$t_{1}, t_{2}$ - accordingly, the temperature of heating of $R 1, R 2$ thermoresistive elements;

$\alpha$ - temperature coefficient of material resistance of the thermoresistive elements.

The $\Delta R 4$ resistance increase does not depend on the $t$ initial temperature, and the scheme's operation is independent of the ambient temperature. The temperature difference between the thermistor elements is due to the $P_{1}$ and $P_{2}$ capacities. Thermal resistor elements are connected in series $(R 2=4 R 1)$, the following (7) dependencies of capacities are obtained.

$$
P_{1}=I \cdot U / 5=P / 5 \text { i } P_{2}=4 \cdot I \cdot U / 5=4 \cdot P / 5,
$$

where $P$ - power coming from the rectangular oscillator generator;

$U$ - voltage amplitude;

$I$ - amperage.

The values of the $\Delta t_{1}$ and $\Delta t_{2}$ temperatures for the overheating of the sensors are proportional to the given power and with the (8) dependencies are described.

$$
\Delta t_{1}=\frac{P_{1}}{\beta \cdot S_{e t}}=\frac{P}{5 \cdot \beta \cdot S_{e t}}, \Delta t_{2}=\frac{P_{2}}{\beta \cdot S_{e t}}=\frac{4 \cdot P}{5 \cdot \beta \cdot S_{e t}},
$$

where $\beta$-heat-transfer coefficient of the sensors, $\beta=\beta_{0}+K \cdot v^{n}$;

$\beta_{0}$ - heat-transfer coefficient of the thermistor elements at the milk ejection absence (rate of movement $v=0$ );

$K$ - aspect ratio.

Taking into account the equations (6)-(8), the dependence (9) is obtained.

$$
\Delta R 4=\frac{3 \cdot R 3 \cdot \alpha \cdot R 1_{0} \cdot R 2_{0}}{5 \cdot R 1^{2} \cdot \beta \cdot S_{e t}} \cdot P .
$$

The value $\Delta R 4$ is set at the time of adjustment of the bridge circuit of the meter and in the automatic mode of operation is constant. Taking into account the equation (9) and the dependence of the coefficient of heat transfer, the power of measurement of milk ejection (12) is obtained.

$$
P=\frac{5 \cdot R 1^{2} \cdot \Delta R 4 \cdot S_{e t}}{3 \cdot R 3 \cdot \alpha \cdot R 1_{0} \cdot R 2_{0}} \cdot\left(\beta_{0}+K \cdot v^{n}\right) .
$$

Taking into account that the exponent $n<1$ the equation (12) is differentiated and depending on the velocity of the milk in the sensor, the differential sensitivity (13) of the scheme is determined.

$$
\frac{d P}{d v}=\frac{5 \cdot R 1^{2} \cdot \Delta R 4 \cdot S_{e t}}{3 \cdot R 3 \cdot \alpha \cdot R 1_{0} \cdot R 2_{0}} \cdot \frac{n}{v^{1-n}} .
$$

Power $P$ of the bridge circuit in the pulse mode is given as the average value for the time interval of both the $\Delta \tau_{H}$ heating and $\Delta \tau_{0}$ cooling of the elements (Fig. 2, b, d) (14).

$$
P=\frac{1}{\Delta \tau_{H}+\Delta \tau_{o}} \cdot\left(P_{H} \cdot \Delta \tau_{H}+P_{o} \cdot \Delta \tau_{o}\right),
$$

where $P_{H}$ - output power of the 10 generator of rectangular oscillations at the amplitude of the output voltage of the heating $U_{H}$ (fig. 2,d);

$\Delta \tau_{H}$ - duration of heating of thermistor elements during one cycle of automatic operation of the circuit (heating-cooling) (fig. 2, b, d);

$P_{O}$ - output power of the generator of rectangular oscillations 10 at the amplitude of the output voltage $U_{O}$ (fig. 2, d) in the mode of "cooling" of the elements;

$\Delta \tau_{0}$ - duration of "cooling" of the thermistor elements.

During the $\Delta \tau_{H}$ time through the 5 electronic key from the 9 high frequency pulse generator the (15) number of impulses $N_{1}$ will go to the bus-bar former with the $\Delta \tau_{h f p}$ time interval among impulses.

$$
N_{i}=\Delta \tau_{H} / \Delta \tau_{h f p} .
$$


Equations of (15), (14) and (12) are equated and after changes the (16) dependence is obtained.

$$
N_{i}=\frac{\left(A \cdot \beta_{o}-P_{o}\right) \cdot \Delta \tau}{\Delta \tau_{h f p} \cdot\left(P_{H}-P_{o}\right)}+\frac{A \cdot K \cdot \Delta \tau}{\Delta \tau_{h f p} \cdot\left(P_{H}-P_{o}\right)} \cdot v,
$$

where $A=\frac{5 \cdot R 1^{2} \cdot \Delta R 4 \cdot S_{e t}}{3 \cdot R 3 \cdot \alpha \cdot R 1_{0} \cdot R 2_{0}}$

$\Delta \tau=\Delta \tau_{H}+\Delta \tau_{o}$, or (from the fig. 2 , c, d) $\Delta \tau=\tau_{i}-\tau_{i-1}$ - time interval of one cycle of operation of the circuit (heating-cooling of thermoresistor elements).

The first term of (16) equation is constant and with the selection of the electric circuit ratings can be compensated at the condition of $P_{o}=A \cdot \beta_{0}$. Then, at the output of the bus-bar former, the function of the number of pulses is obtained, which characterizes the milk output for the $\Delta \tau$ time interval, and after multiplication and division into $S_{s k}$ the dependence (17) is obtained.

$$
N_{i}=\frac{A \cdot K}{\Delta \tau_{h f p} \cdot\left(P_{H}-P_{o}\right) \cdot S_{s k}} \cdot S_{s k} \cdot \Delta \tau \cdot v
$$

Product of $S_{s k} \cdot v \cdot \Delta \tau=q_{f . i}$ characterizes the milk output for the $\Delta \tau_{i}$ time interval.

\section{Results and discussion}

The results of the research showed that when the bridge scheme of the thermistor sensors with a voltage of $4 \mathrm{~V}$ is powered, the duration of the $\Delta \tau_{H}$ heating mode is $49.6 \mathrm{~ms}$ for one work cycle of the scheme $(\Delta \tau)$ at the intensity of $q=0.39 \mathrm{~kg} \cdot \mathrm{min}^{-1}$ milk ejection, and with milk ejection of $q=3.042 \mathrm{~kg} \cdot \mathrm{min}^{-1}$, the $\Delta \tau_{H}=215.8 \mathrm{~ms}$ accordingly. The nature of the change in power consumption of the measurement scheme of the intensity of milk ejection is nonlinear (Fig. 3) at $4 \mathrm{~V}$ supply voltage of the bridge circuit. An increase in the $U_{H}$ supply voltage of the bridge circuit leads to an increase in the $U$ output voltage of the bridge circuit and its sensitivity accordingly (Fig. 4). The AC frequency of the bridge circuit supply is $15 \mathrm{kHz}$. The dependence between the $N_{i}$ number of pulses and the intensity of milk ejection $q$ has a nonlinear characteristic (Fig. 5).

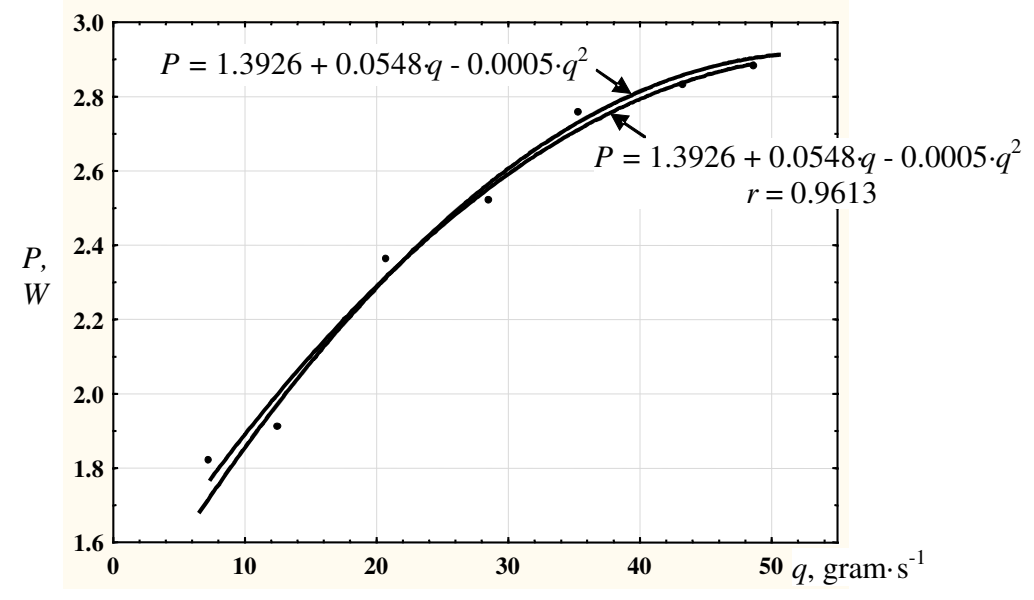

Fig. 3. Dependence of thermoanemometric sensor $P$ measurement power on intensity of $q$ milk ejection 


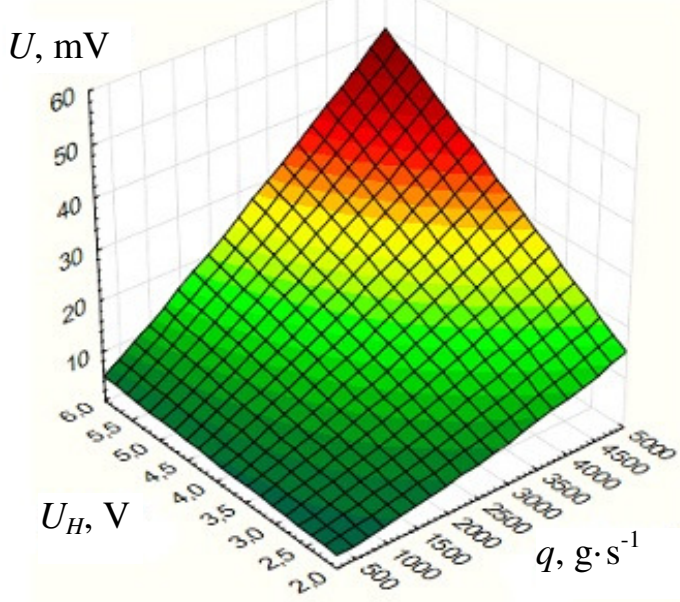

Fig. 4. Dependence of $\boldsymbol{U}$ output voltage from $\boldsymbol{U}_{\boldsymbol{H}}$ supply voltage of bridge circuit and intensity of $q$ milk ejection

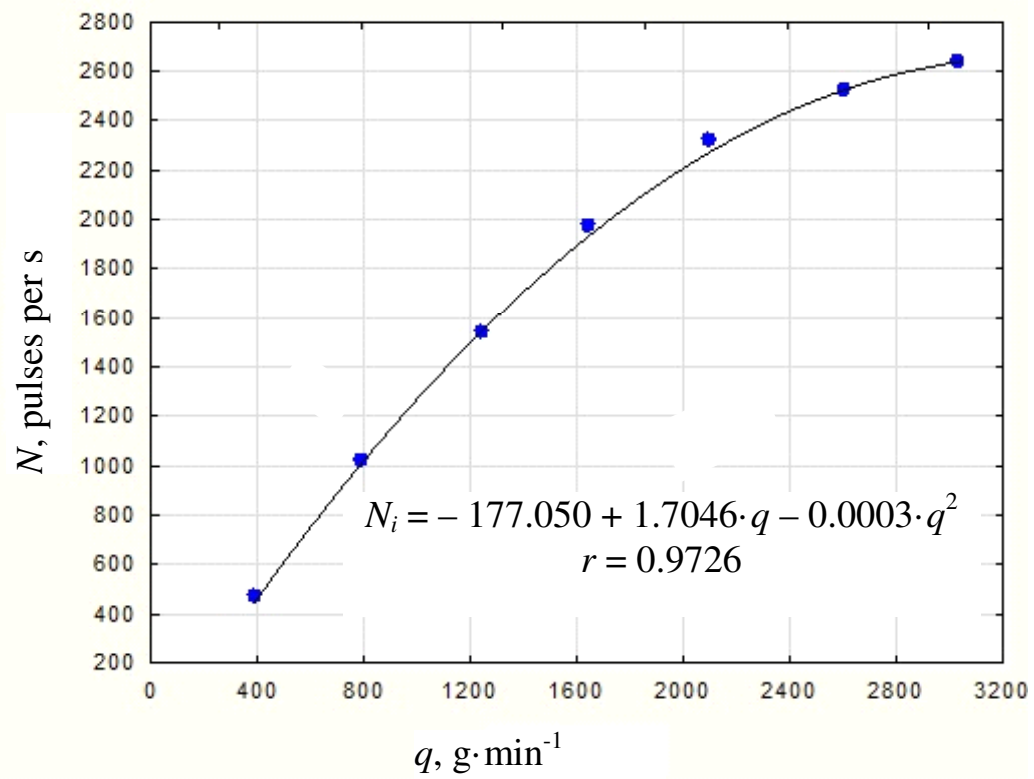

Fig. 5. Dependence of $N_{i}$ number of impulses of measurement scheme of thermoanemometric measuring device on intensity of $q$ milk ejection

The high-frequency pulse generator operates at the frequency of $12 \mathrm{kHz}$, and the $\Delta \tau_{h f p}=83.3 \mu \mathrm{s}$ accordingly. The time interval of one work cycle of "heating-cooling" of the thermistor elements circuit is $\Delta \tau=1 \mathrm{~s}$.

At small milk ejection, the self-descriptiveness of the measuring device is higher, which is important for automation of the technological process of cow machine milking.

\section{Conclusions}

1. The analysis of the differential sensitivity of the power of milk ejection measuring shows that for $n<1$ the differential sensitivity of the measurement scheme is increased with the decrease in the $v$ velocity. The bridge circuit should be adjusted to operate on an initial, near-linear section of the characteristics of the thermistor elements.

2. The optimum power of the bridge circuit supply is within the range of $6-10 \mathrm{~W}$ at the milk ejection measurement power (the power that is taken off by the milk flow during the milking process) of $1.5-4 \mathrm{~W}$, at the bridge circuit powered with a pulse voltage of $4 \mathrm{~V}$ and the frequency of $15 \mathrm{kHz}$. 
3. Using a discrete digital signal to measure milk ejection provides the required precision and simplifies the conversion of "digital code - intensity of milk ejection" and also facilitates the counting of the milk quantity.

\section{References}

[1] Baker R. C. Flow measurement handbook: industrial designs, operating principles, performance, and applications. Cambridge University Press, New York, 2000. 524 p.

[2] Hardy J. E., Hylton J. O., McKnight T. E. Empirical correlations for thermal flowmeters covering a wide range of thermal-physical properties. In: National Conference of Standards Labs (NCSL), 1999. [online] [30.01.2018]. Available at: http://www.iaea.org/inis/collection/NCL CollectionStore/Public/30/048/ 30048008.pdf.

[3] Olin J. G. Industrial Thermal Mass Flowmeters, Part 1: Principles of Operation. Measurements and Control, vol. 193, 1999. 16 p. [online] [30.01.2018]. Available at: http://www.sierrainstruments.com/prnews/mnc.pdf.

[4] Badarlis A., Kumar V., Pfau A., Kalfas A. Novel sensor geometry for liquids serving in dispersion thermal flow meters. In: SENSOR+TEST Conferences 2011 - SENSOR Proceedings, 2011, pp. 78-83.

[5] Baker R.C., Gimson C. The effects of manufacturing methods on the precision of insertion and inline thermal mass flowmeters. Flow Measurement and Instrumentation, 12, no. 2, 2001, pp. 113-121.

[6] The effect of gas properties and installation effects on thermal mass flowmeters. Report no. 2002/53, 2003. [online] [30.01.2018]. Available at: http://www.tuvnel.com/_x90lbm/ Report_FDMS03.pdf.

[7] Klemen Rupnik, Jože Kutin, Ivan Bajsić. A Method for Gas Identification in Thermal Dispersion Mass Flow Meters. Strojniški vestnik - Journal of Mechanical Engineering, 60/9, 2014, pp. 607 616. [online] [30.01.2018]. Available at: http://en.sv-jme.eu/data/upload/2014/09/08_2014_ 1889_Rupnik07.pdf

[8] Artur Cebula. Experimental and numerical investigation of thermal flow meter. Archives of thermodynamics, 36/3, 2015, pp. 149-160. [online] [30.01.2018]. Available at: http://at.czasopisma.pan.pl/images/data/ at/wydania/No_3_2015/aoter-2015-0027.pdf

[9] Farzaneh-Gord M., Parvizi S., Arabkoohsar A., Machado L., Koury R. Potential use of capillary tube thermal mass flow meter to measure residential natural gas consumption. J. Nat. Gas Sci. Eng. 22, 2015, pp. 540-550

[10] Dmytriv V., Dmytriv I. Thermo-anemometer measuring of two-phase pulsating flows applying to the cyber-physical system of milk production. V International Scientific Congress "Agricultural Machinery 2017”. Varna, BULGARIA, Year I, Vol. 1/1, 2017, pp. 85-87. [online] [30.01.2018]. Available at: http://www.agrimachinery.net/sbornik/1-2017.pdf. 